POLIBUETÁNICA

Núm. 40, pp. 109-125 México, 2015

DOI: $10.18387 /$ polibotanica.40.7

\title{
ANÁLISIS CARIOTÍPICO DE AGAVE MARMORATA Y AGAVE PEACOCKII (AGAVACEAE) UBICADOS EN LAS TERRAZAS ALUVIALES DEL RÍO ZAPOTITLÁN, PUEBLA, MÉXICO
}

\section{KARYOTYPE ANALYSIS OF AGAVE MARMORATA AND AGAVE PEACOCKII (AGAVACEAE) FROM ALLUVIAL TERRACES OF THE ZAPOTITLÁN RIVER, PUEBLA, MEXICO}

\author{
Saúl Flores-Maya ${ }^{1}$, Miguel Ángel Vargas-Juradoํㅜ, \\ Mario Ernesto Suárez-Mota ${ }^{1}$ y Héctor Barrera-Escorcia ${ }^{2}$
}

Facultad de Estudios Superiores Iztacala. Av. de los Barrios, Núm.1 Los Reyes Iztacala, Tlalnepantla, Estado de México, México, CP 54090. Teléfono: 5556231134. ${ }^{1}$ Laboratorio de Recursos Naturales UBIPRO, ${ }^{2}$ Laboratorio de Microscopia UNAM-FESI. Correoelectrónico: saulsel@unam.mx; tonatiu969@hotmail.com; hectorbarrerae@hotmail.com

\section{RESUMEN}

El objetivo de este estudio fue determinar la distribución espacial, el número cromosómico y el cariotipo de Agave marmorata Roezl y Agave peacockii Croucher dentro del Valle de Zapotitlán Salinas, Puebla. Este estudio reporta por primera vez el número cromosómico de A. marmorata y A. peacockii, cuyos niveles de ploidía en ambas especies es de $2 \mathrm{n}=2 \mathrm{x}=60$. Por otra parte, la distribución de A. marmorata en el área estudiada es más amplia respeto de $A$. peacockii que únicamente se la encuentra al suroeste. La distribución espacial de $A$. peacockii está limitada por la variación en las condiciones del suelo, los tipos de vegetación de la zona, por su condición de diploide (2x) y su posible carácter híbrido, por lo tanto, su presencia en la zona depende de la sincronización de la floración de sus progenitores y su capacidad de reproducción sexual y asexual.

Palabras clave: asimetría cariotípica, Valle de Tehuacán-Cuicatlán, Ortoselección cariotípica
The aim of this study was to determine the spatial distribution, chromosome number and karyotype of Agave marmorata Roezl and Agave peacockii Croucher within Zapotitlan Salinas Valley, Puebla. This study reports for the first time the chromosome number of A. marmorata and A. peacockii, which shows that the ploidy levels of both species is $2 \mathrm{n}=2 \mathrm{x}=60$. Moreover, the distribution of $A$. marmorata in the area is larger that, A. peacockii species only placed at southwestern zone. In conclusion, the spatial distribution of $A$. peacockii is limited by variation in soil conditions, vegetation types of the area, for being a diploid $(2 \mathrm{x})$ and their possible hybrid nature, so their presence in the area depends on the timing the flowering of the parents and their ability to reproduce, by sexual and asexual means.

Key words: karyotype asymmetry, Tehuacan-Cuicatlan Valley, orthoselection. 


\section{INTRODUCCIÓN}

La familia Agavaceae es nativa de América. En la actualidad, ocho géneros son reconocidos, todos los miembros de esta familia presentan una estructura básica en cuanto a sus rosetas, flores e inflorescencias (García-Mendoza, 1995).

El género Agave incluye alrededor de 200 especies y el $75 \%$ de éstas tiene como centro de origen a México, (Gentry, 1982; Reinchenbacher, 1985; Álvarez de Zayas, 1989; García-Mendoza, 2007). De las especies restantes algunas de ellas se ubican en el sur de los Estados Unidos de América con aproximadamente 15 especies, principalmente en Arizona; 12 en las Antillas (Álvarez de Zayas, 1989), y el resto en América central y el norte de Sudamérica (por ejemplo en Bolivia y Paraguay).

La mayor diversidad del género Agave se encuentra en el centro de México, en el Valle de Tehuacán-Cuicatlán, en el cañón barranca de Meztitlán, La Sierra Madre Occidental y en las regiones del desierto de Chihuahua, el cual podría ser el centro del origen del género. Un hecho importante es que la mayoría de las especies del género Agave son endémicas y sólo son encontradas en pocos hábitats específicos y montañas (García Mendoza, 2002, 2007).

Agave marmorata Roezl (Agave todaroi Baker) es una especie endémica de México. Su distribución abarca todo el Valle de Tehucán-Cuicatlán. El nombre común con el cual se conoce localmente es "pitzometl o "pichomel", "huiscole" o "maguey curandero". El último nombre hace referencia al uso medicinal que le dan los habitantes de la localidad. Es una especie con rosetas largas, tallo corto, despliega de 30-50 hojas, $1.2 \mathrm{~m}$ de alto, $2 \mathrm{~m}$ de ancho, con pequeñas flores amarillas brillantes de $48 \mathrm{~mm}$ de longitud. Posee generalmente espinas cónicas de aproximadamente $1.5-3 \mathrm{~cm}$. Los ovarios presentes en las hojas alcanzan magnitudes entre 20-25 mm (Gentry, 1982).

Agave peacockii Croucher (Agave roezliana var. peacockii; Agave ghiesbreghtii var. peacockii) es una especie endémica de México y en especial del valle de Zapotitlán. El nombre común con el cual se le conoce en Zapotitlán Salinas es "Cateshe" y "Capulixtle". Es de tamaño mediano a largo con rosetas abiertas que se separan con largas hojas armadas de espinas y racimos libres en el que se observan las inflorescencias. En etapa madura presenta de 60-145 hojas que miden de 10-16 cm, lineales o lanceoladas, usualmente pareadas, cercanas al centro, rígidas y con ascensión horizontal. Las espinas poseen longitudes de $2.5-9 \mathrm{~cm}$, aciculares y presenta una coloración café oscuro a gris. Además, en la parte interior de las hojas posee protuberancias a manera de espinas. Las inflorescencias llegan a medir 3-5 m. Los ovarios miden unos 24-28 cm (fig. 1) (Gentry, 1982).

A pesar del valor cultural, ornamental, económico y biológico que representa este grupo de plantas son pocos los estudios citogenéticos que se registran en la literatura científica. Sin embargo, como lo señalaron Castorena-Sánchez (1985) y Castorena-Sánchez et al. (1991), es el interés de estudiar.

La citogenética clásica de este género podría conducir a un mejor entendimiento de su biología en el aspecto genético y aportar bases científicas para dilucidar su taxonomía. Con base en lo anterior se justifica el llevar 


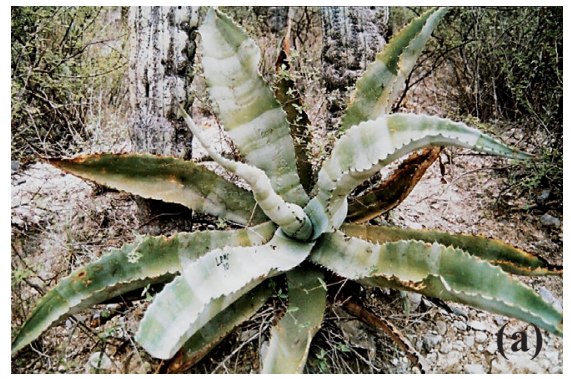

Fig. 1. Agave marmorata (a) y Agave peacockii (b). a cabo estudios de citogenética básica para establecer características como el número cromosómico y cariotipo presente en las diversas especies de este género a lo largo de su distribución.

Durante el tiempo de desarrollo de este trabajo no se encontraron referencias sobre los reportes del número cromosómico de Agave marmorata y Agave peacockii.

En la base de datos del Index to plant Chromosome Numbers (IPCN) del Jardín Botánico de Missouri se establecen 31 reportes del número cromosómico del género Agave, los cuales señalan la condición $2 \mathrm{x}, 3 \mathrm{x}$ y $4 \mathrm{x}$ del género (IPCN, 2013).

Uno de los primeros estudios realizados sobre el género Agave es de Granik (1944), el cual presenta el número cromosómico de 30 especies de magueyes; con el cual se pudo determinar la serie euploide de $2 \mathrm{x}, 3 \mathrm{x}$, $4 x, 5 x$ y $6 x$ del género.

Castorena-Sánchez et al. (1991) realizaron un estudio para establecer el número cromosómico, el cariotipo y variación cromosómica natural de $A$. angustifolia, $A$. angustifolia var. marginata, A. fourcroy-



des, A. sisalana, A. tequilana y un híbrido 11648 , en donde los números cromosómicos reportados para cada especie de maguey fueron: $2 \mathrm{n}=6 \mathrm{x}=180,2 \mathrm{n}=2 \mathrm{x}=60,2 \mathrm{n}=5 \mathrm{x}=150$, $2 \mathrm{n}=5 \mathrm{x}=150,2 \mathrm{n}=2 \mathrm{x}=60$ y $2 \mathrm{n}=2 \mathrm{x}=60$, respectivamente.

Flores-Maya et al. (2010) realizaron un estudio citogenético dentro del valle de Zapotitlán Salinas, Puebla, en A. karwinskii Zucc. y A. macroacantha Zucc., donde los conteos cromosómicos reportados muestran que en toda la zona de estudio se presenta un único nivel de ploidía $(2 n=2 x=60)$. En la actualidad las conclusiones de investigaciones, que se iniciaron desde 1944, sostienen que los datos cromosómicos característicos para la familia Agavaceae muestran que el número básico (x) y el haploide es $30(2 \mathrm{n}=2 \mathrm{x}=60)$ (Granick, 1944; Cave, 1964; Bhattacharyya, 1968; Vázquez, 1977; Pinkava y Baker, 1985; Pinkava et al., 1992; Martínez-Palacios et al., 1999). Las especies de Agave se caracterizan por tener cariotipos bimodales altamente asimétricos tanto en su condición diploide como poliploide (Castorena-Sánchez et al., 1991). Este rasgo bimodal está dado por un grupo de cinco pares de cromosomas grandes y veinticinco pequeños, lo cual sugiere que evolutivamente existe una 
tendencia a la ortoselección cariotípica. Este proceso podría consistir en la amplificación de regiones de $\mathrm{ADN}$ que no codifican genes, en sitios distribuidos entre las regiones de entrecruzamiento en los cromosomas del complemento (Brandham y Doherty, 1998). Este proceso da como resultado un número básico uniforme y cariotipos que mantienen su estructura con cromosomas grandes y pequeños (Brandham y Doherty, 1998; Flores-Maya et al., 2010).

Por último, Valverde et al. (1996), hicieron un análisis morfométrico de $A$. marmorata y A. kerchovei sugiriendo que $A$. peacockii es un híbrido de estas dos especies, hecho que ya previamente había sido insinuado Gentry (1982) basándose en sus características somáticas.

A. marmorata y A. peacockii son especies endémicas del Valle de Tehuacán-Cuicatlán. Esta reserva ecológica está sometida a un proceso de desertificación y desertización, presenta baja productividad, hay una disminución y pérdida de la biodiversidad, así como un nivel socioeconómico bajo. (SEMARNAT, 2005).

Por lo mencionado anteriormente, la zona tiene gran importancia para los estudios ecológicos y citogenéticos en magueyes, principalmente porque constituye una de las áreas que comprende la mayor riqueza y número de endemismos de estas plantas a nivel mundial y nacional (García-Mendoza, 2007; CONABIO, 2012). Esta investigación tiene como objetivo: realizar los estudios citogenéticos de Agave marmorata Roezl y Agave peacockii Croucher distribuidos en las terraza aluviales del Valle de Zapotitlán Salinas, Puebla.

\section{MATERIAL Y MÉTODOS}

\section{Área de estudio}

La región de Zapotitlán es un ecosistema semiárido localizado en la región meridional suroeste del estado de Puebla y noreste del estado de Oaxaca en México, entre los $18^{\circ} 12^{\prime}$ y $18^{\circ} 24^{\prime}$ de latitud Norte y entre los $-97^{\circ} 24^{\prime}$ y $-97^{\circ} 36^{\prime}$ de longitud Oeste. Presenta una precipitación anual de aproximadamente $400 \mathrm{~mm}$ y una temperatura de entre $18-22{ }^{\circ} \mathrm{C}$ (Zavala-Hurtado, 1982). Según Rzedowski (1978), el tipo general de vegetación es matorral xerófito, conformado por una vegetación adaptada a ambientes áridos y caracterizada por numerosos taxones, principalmente arbustivos espinosos, herbáceas anuales y suculentas con formas de vida muy variadas (cactáceas, agaváceas, bromeliáceas). El área es parte de la provincia florística del Valle de Tehuacán-Cuicatlán, el cual ha mostrado un nivel significativo de diversidad florística y endemismos. El clima general de Zapotitlán Salinas se caracteriza por ser seco con lluvias en verano, con dos máximos de lluvia en junio y septiembre; separados por dos estaciones secas (ValienteBanuet et al., 1995).

La parte baja de la cuenca del río Salado o Zapotitlán ha sido seleccionada porque en ésta se encuentra una unidad geomorfológica formada de terrazas aluviales, constituidas de sedimentos transportados de diferentes orígenes que han rellenado las partes bajas del valle, formando así suelos profundos que sirven de soporte para el desarrollo de comunidades vegetales conocidas como mezquitales (INEGI, 1987; Barrera, 2001; García, 2001; López-Galindo et al., 2003). 
El estudio se realizó en cuatro zonas de las terrazas aluviales que fueron establecidas de acuerdo con Oliveros-Galindo (2000; fig. 2). Estos sitios presentan diversos gradientes de perturbación en el suelo, en la estructura y la diversidad de la vegetación (Oliveros-Galindo, 2000; Galindo et al., 2007). La zona A es un sitio poco alterado. Los perfiles y horizontes del suelo están completos y bien desarrollados, el suelo es franco-arenoso. La vegetación muestra una estructura y una fisonomía bien definidas, con una riqueza de especies y con un patrón claro de estratificación vertical. En contraste, la zona $\mathrm{D}$ se considera muy deteriorada, porque el suelo original ha desaparecido, mostrando sedimentos arcillosos y derivados de lutita y caliza. Las zonas B y C son sitios también muy perturbados, aquí prácticamente la vegetación original del tipo de matorral xerófilo, está siendo eliminada (fig. 2) (Galindo et al., 2007).

Las zonas de muestreo en las terrazas aluviales ocupan una extensión total de $9600 \mathrm{~m}^{2}$.

\section{Muestras biológicas}

Se realizaron recorridos en la zona de estudio, en los cuales se colectaron hijuelos de cada una de las especies (plantas de origen asexual) y se georeferenciaron con un GPS (Garmin's $12 \mathrm{XL}$ ); y con cartas topográficas digitalizadas (INEGI, 2005) se determinó su distribución espacial en el municipio de Zapotitlán Salinas (fig. 2). Lo anterior permitió realizar una selección de cuatro diversos sitios de muestreo, colectando dos hijuelos de cada especie en cada punto.

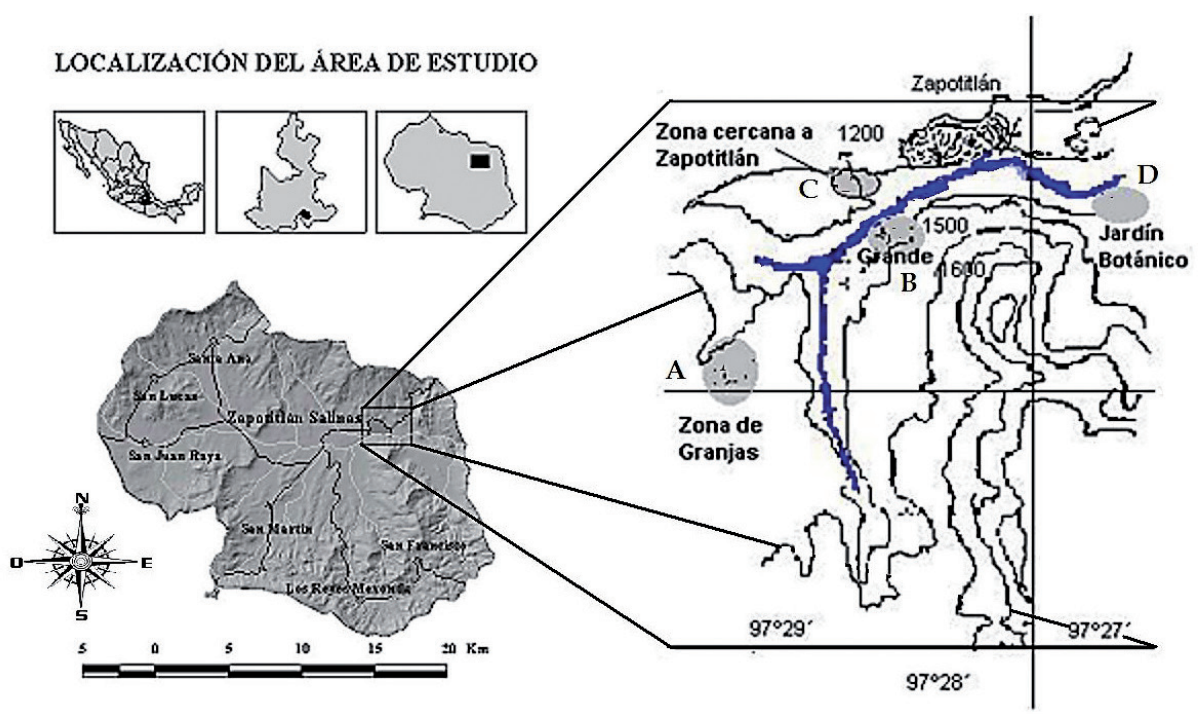

Fig. 2. Ubicación geográfica de Zapotitlán Salinas, Puebla, México, indicando las zonas de muestreo (tomado de Oliveros-Galindo, 2000). 


\section{Estudio citogenético}

Los hijuelos colectados sirvieron para el estudio citogenético, el cual se realizó en los meristemos radiculares de las especies vegetales de acuerdo con el método propuesto por Rodríguez y Seijo (2001) y García (1990). El conteo cromosómico se realizó en 10 individuos de cada especie. Para ambos casos, las medidas de los cromosomas fueron estimadas a partir de tres placas en metafase por individuo. La elaboración de las laminillas consistió en lo siguiente: a cada una de las especies se les determinó la hora mitótica. Una vez establecida la hora mitótica, a 10 meristemos, se procedió al bloqueo de la metafase con 8-hidroxiquinoleina $0,002 \mathrm{M}$, por un tiempo de cuatro horas. Se cortaron las raíces y se fijaron en solución Farmer 3:1 (alcohol etílico-ácido acético glacial) durante 24 horas, luego fueron tratadas con ácido clorhídrico $1 \mathrm{~N}$ durante cinco minutos para su hidrólisis a una temperatura de $55^{\circ} \mathrm{C}$. La tinción de los cromosomas fue efectuada con aceto-orceína al 1\%. Para determinar el número cromosómico de las especies mencionadas, se analizaron las diez mejores células por especie que mostraron claramente la morfología de los cromosomas.

\section{Análisis estadísticos}

El resultado de los conteos cromosómicos de cada una de las especies en estudio fue ordenado en tablas y se calcularon las medias para el número cromosómico diploide, así como las desviaciones estándar y varianzas respectivas. Se calculo la media poblacional y a partir de estos valores promedio se utilizó el estadístico de prueba " $t$ " student para asegurase que las muestras tomadas pertenecen a la misma especie, (Sokal y Rohlf, 1995). Estos datos junto con los re- sultados ecológicos se complementaron para la formación del banco de datos biológicos de la zona.

Para la elaboración de los cariogramas se escogieron las mejores células y se obtuvieron una serie de fotografías de figuras en metafase que fueron procesadas por un software de análisis de imagen versión 3.1 (UTHSCSA, 2012).

Se calculó la media de la longitud de cada uno de los brazos cromosómicos y la longitud total de los pares cromosómicos, la longitud relativa, y el índice centromérico. Con el índice centromérico se realizó la clasificación de los cromosomas de acuerdo a lo establecido por Levan et al., (1964).

También se definió el grado de asimetría del cariotipo de estas especies basado en las categorías establecidas por Romero-Zarco (1986). Para el coeficiente de asimetría intracromosómico, de acuerdo a Romero-Zarco (1986), se aplicó la fórmula A1 $=1-\Sigma(\mathrm{bi} /$ $\mathrm{Bi}) / \mathrm{n}$; donde: bi, es el promedio del tamaño para brazos cortos de cada cromosoma homólogo y Bi es el promedio de tamaño para el brazo largo en cada par cromosómico homólogo. Para la variación de asimetría intercromosómico se usó la fórmula del coeficiente de dispersión de Pearson, que es la proporción entre la desviación estándar y la media de la longitud de los cromosomas para cada muestra: $\mathrm{A} 2=\mathrm{s} / \mathrm{x}$.

\section{Estudio biogeográfico}

Para evaluar la distribución de las especies en un espacio geográfico se hizo un análisis de su distribución geográfica conocida en el Valle de Tehuacán-Cuicatlán (VTC), particularmente en el municipio de Zapotitlán 
(figs. 5 y 6). Con los registros de las especies y empleando el programa de máxima entropía (MaxEnt 3.3.3e) se realizaron los modelos de la distribución potencial de las especies y se verificó su correspondencia con los tipos de vegetación que se encuentran en el VTC. Estos tipos de vegetación se delimitaron geográficamente con el mapa de la serie III de INEGI (INEGI, 2005). El manejo y análisis de estos datos se realizó empleando un sistema de información geográfica ( SIG; ArcView 3.2) (ESRI, 2000). Este SIG cuenta con una aplicación (CATS) diseñada por la Unión Mundial para la Conservación de la Naturaleza (UICN) que considera que una buena estrategia de análisis de los patrones de distribución de las especies es mediante el uso de cuadros o celdas en que se divide la región de estudio (UICN, 2001). El tamaño de la cuadrícula en que se dividió el VTC fue determinado siguiendo las recomendaciones de la misma IUCN. En este trabajo se calculó la distancia de los sitios extremos conocidos para cada especie; tomando el $10 \%$ de la distancia extrema de cada especie y promediando todos los datos se obtuvo un valor que fue utilizado para determinar el tamaño de celda $\left(1 \mathrm{~km}^{2}\right)$ (Suárez-Mota y Villaseñor, 2011; Suárez-Mota et al., 2013). Con esta misma aplicación se obtienen datos con base en la definición de áreas de ocupación propuesta por Rapoport (1975) a partir de análisis areográficos.

\section{RESULTADOS}

\section{Estudio citogenético}

Los resultados obtenidos en el índice mitótico de Agave marmorata para localizar el mayor número de metafases fue a las 9:00 AM. Por otra parte, Agave peacockii mostró dos horas de colecta para localizar las células en metafases, éstas fueron a las 6:00 AM y a las 15:00 PM (fig. 3).

Basándose en el análisis de la prueba de " $t$ " de student $(\mathrm{p}<0.05)$ de los conteos cromosómicos de cada una de las especies, y en cada zona, se determinó que los datos pertenecían a la misma población y que el número cromosómico fue $2 \mathrm{n}=2 \mathrm{x}=60$ para A. marmorata al igual que para $A$. peacockii (tabla 1). El número cromosómico obtenido para las dos especies coincide con reportes de la literatura para el género Agave. Además se puede observar un rasgo distintivo en el cariotipo de estas especies que es la presencia de cinco pares de cromosomas largos y 25 pares de medianos a pequeños (fig. 4). La clasificación de los cromosomas de $A$. marmorata resultó de la siguiente manera: cuatro fueron subtelocéntricos (st), doce metacéntricos región media $(\mathrm{m})$, nueve submetacéntricos (sm), y cinco metacéntricos en el sentido estricto (M) (tabla 2). Por otro lado, en A. peacockii, se reconocen un par t, cuatro pares st, dos pares sm y $23 \mathrm{M}$. Los pares cromosómicos de las dos especies que fueron clasificados en la misma posición centromérica son los pares 10,11,12,13 y 14, es decir, sólo un $8.3 \%$ de su morfología es similar (fig. 4).

En la determinación de la simetría cariotípica el índice de asimetría intracromosomal para $A$. marmorata fue de $\mathrm{A} 1=0.98$ y para A. peacockii fue de $\mathrm{A} 1=0.98$, y el valor del índice intercromosomal para de A. marmorata fue de 0.53 y el de $A$. peacockii fue 0.86 (tabla 2). El estudio areográfico Agave marmorata se distribuye en el área que abarca toda la localidad de Zapotitlán Salinas. Sin embargo, las poblaciones de dicho agave se encuentran aisladas. Por otra parte, Agave 


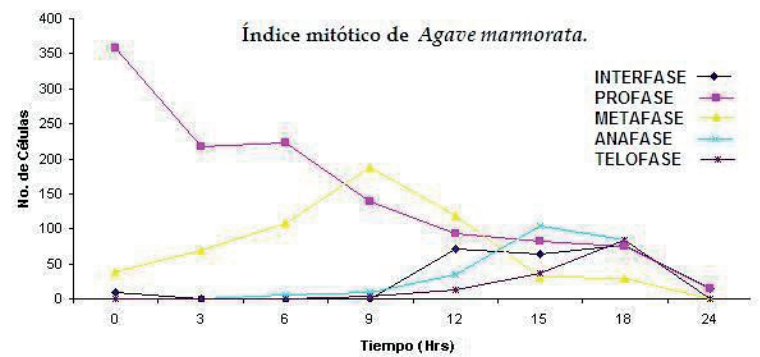

(a)

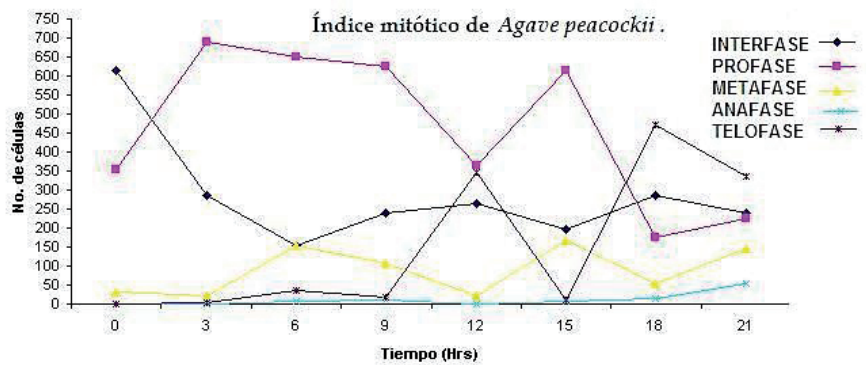

(b)

Fig. 3. Ciclo celular de la zona radicular de Agave marmorata (a) y Agave peacockii (b).

Tabla 1. Puntos de muestreo en las terrazas aluviales del valle de Zapotitlán Salinas, Puebla, México, y número cromosómico obtenido en las plantas en las diferentes zonas.

Comparación entre las medias $(\bar{\chi}) \pm$ la desviación estándar (D.S.).

\begin{tabular}{|c|c|c|c|c|c|c|}
\hline \multicolumn{2}{|c|}{ TAXONES } & \multirow{2}{*}{$\begin{array}{c}\text { Zona A } \\
\text { Las Granjas } \\
\text { 1496-1558 m }\end{array}$} & \multirow{2}{*}{$\begin{array}{c}\text { Zona B } \\
\text { Cerro Grande } \\
\mathbf{1 5 2 8 - 1 5 5 9} \mathbf{~ m}\end{array}$} & \multirow{2}{*}{$\begin{array}{c}\text { Zona C } \\
\begin{array}{c}\text { Zapotitlán } \\
\text { 1457-1494 m }\end{array} \\
59.6 \pm 1.26\end{array}$} & \multirow{2}{*}{$\begin{array}{c}\begin{array}{c}\text { Zona D } \\
\text { Jardín } \\
\text { botánico } \\
\text { 1425-1458 m }\end{array} \\
59.8 \pm 1.47\end{array}$} & \multirow{2}{*}{$\begin{array}{c}\begin{array}{c}\mathbf{2 n} \\
\text { Datos } \\
\text { redondeados }\end{array} \\
60\end{array}$} \\
\hline Agave & $\bar{X} \pm$ D.S. & & & & & \\
\hline marmorata & varianza & 1.51 & 0.5 & 1.6 & 2.1 & \\
\hline \multirow{2}{*}{$\begin{array}{c}\text { Agave } \\
\text { peacockii }\end{array}$} & $\bar{X} \pm$ D.S. & ausente & ausente & ausente & $59.8 \pm 1.4$ & \multirow{2}{*}{60} \\
\hline & varianza & ausente & ausente & ausente & 1.95 & \\
\hline
\end{tabular}

peacockii solamente se encontró al suroeste de dicha localidad (figs.5 y 6; tabla 3 ).

Los parámetros de área calculados con el SIG muestran que la distribución espacial de A. marmorata dentro de la zona de estudio abarca un área de aproximadamente 343 hectáreas y un perímetro de $3928 \mathrm{~m}$, en tanto que $A$. peacockii, posee un área aproximada de 100 hectáreas y un perímetro de $1500 \mathrm{~m}$. (fig. 5 y 6). Los datos obtenidos con aplicación del SIG se indican en la tabla 3, estos valores permitieron establecer una categoría del estatus que tendrían las especies en la 

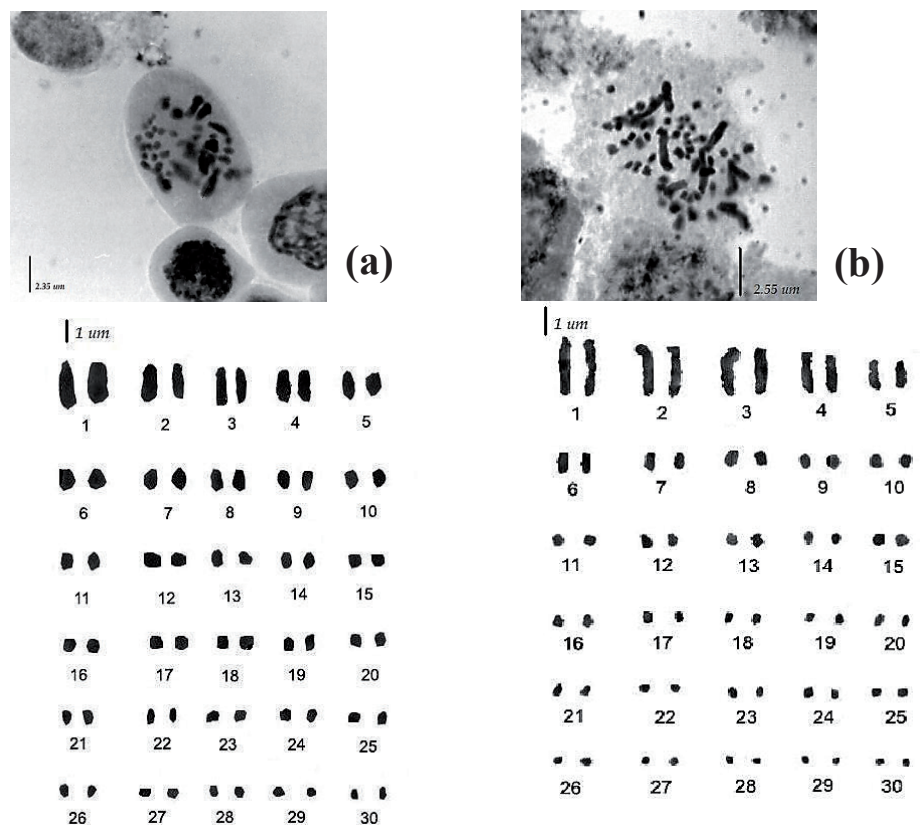

Fig. 4. Célula mitótica y cariograma de Agave marmorata (a) y Agave peacockii (b) con número cromosómico de $2 \mathrm{n}=2 \mathrm{x}=60$. Las barras representan el aumento en micras.

Tabla 2. Caracteres citogenéticos de las especies de Agave estudiadas: número cromosómico $(2 \mathrm{n})$; fórmula cariotípica; longitud total del cariotipo (LTC); longitud total de los brazos cortos ( $\mathrm{LBc}$ ); longitud total de los brazos largos (LBL), y, el tipo de asimetría (A1 y A2) de acuerdo a Romero-Zarco (1986).

\begin{tabular}{|c|c|c|c|c|c|c|c|c|}
\hline \multirow[t]{2}{*}{ Taxones } & \multirow[t]{2}{*}{$2 n$} & \multirow[t]{2}{*}{$\begin{array}{c}\text { Fórmula } \\
\text { cariotípica }\end{array}$} & \multirow[t]{2}{*}{$\begin{array}{c}\text { Valor de la } \\
\text { longitud } \\
\text { total del } \\
\text { complemento } \\
\text { cromosómico } \\
(\text { um }) \\
\end{array}$} & \multicolumn{3}{|c|}{$\begin{array}{c}\bar{X} \pm \text { D.S. } \\
\text { Longitud media de } \\
\text { los cromosomas } \\
\text { (um) }\end{array}$} & \multicolumn{2}{|c|}{$\begin{array}{c}\text { Asimetría } \\
\text { cariotípica }\end{array}$} \\
\hline & & & & LTC & LTBc & LTBL & A1 & $\mathbf{A 2}$ \\
\hline $\begin{array}{c}\text { Agave } \\
\text { marmorata }\end{array}$ & $2 x=60$ & $5 \mathrm{M}, 12 \mathrm{~m}, 9 \mathrm{sm}, 4 \mathrm{st}$ & 267.58 & $0.89 \pm 0.48$ & $0.29 \pm 0.18$ & $0.60 \pm 0.51$ & 0.98 & 0.53 \\
\hline $\begin{array}{c}\text { Agave } \\
\text { peacockii }\end{array}$ & $2 x=60$ & $23 \mathrm{M}, 2 \mathrm{sm}, 4 \mathrm{st}, 1 \mathrm{t}$ & 227.91 & $0.75 \pm 0.66$ & $0.25 \pm 0.16$ & $0.49 \pm 0.5$ & 0.98 & 0.86 \\
\hline
\end{tabular}


Tabla 3. Datos obtenidos con los análisis areográficos.

\begin{tabular}{|l|l|l|}
\hline Parámetros & Agave marmorata & Agave peacockii \\
\hline Número de registros (localidades) & 11 & 5 \\
\hline Área de ocupación km² & 16.15 & 296.64 \\
\hline Diámetro máximo (km) & 16.27 & 43.43 \\
\hline Celdas de $1 \mathrm{~km}^{2}$ con registros & 5 & 5 \\
\hline Área de las celdas con registros km² & 5 & 5 \\
\hline Subpoblaciones & 4 & 5 \\
\hline Área (Rapoport) km² & 0044.92 & 1494.66 \\
\hline Subpoblaciones (Rapoport) & 2 & 2 \\
\hline Estatus & en peligro (CR) & en peligro (EN) \\
\hline
\end{tabular}

lista roja de la UICN. Sin embargo, estos datos podrán corroborarse con trabajo de campo aplicando métodos enfocados en la conservación de especies que permiten establecer dichos estatus adecuadamente.

Los modelos de distribución potencial generados indican las posibles áreas que estas especies de Agave pudieran ocupar dentro del VTC y su expansión hacia otras regiones del país. En el caso de $A$. marmorata el modelo señala que esta especie podría extenderse hacia el norte de México mientras que $A$. peacockii tiende a encontrar afinidad hacia la parte del suroeste (figs. 5 y 6 ).

Con relación en el tipo de suelo, se observó que $A$. marmorata tiene mayor preferencia
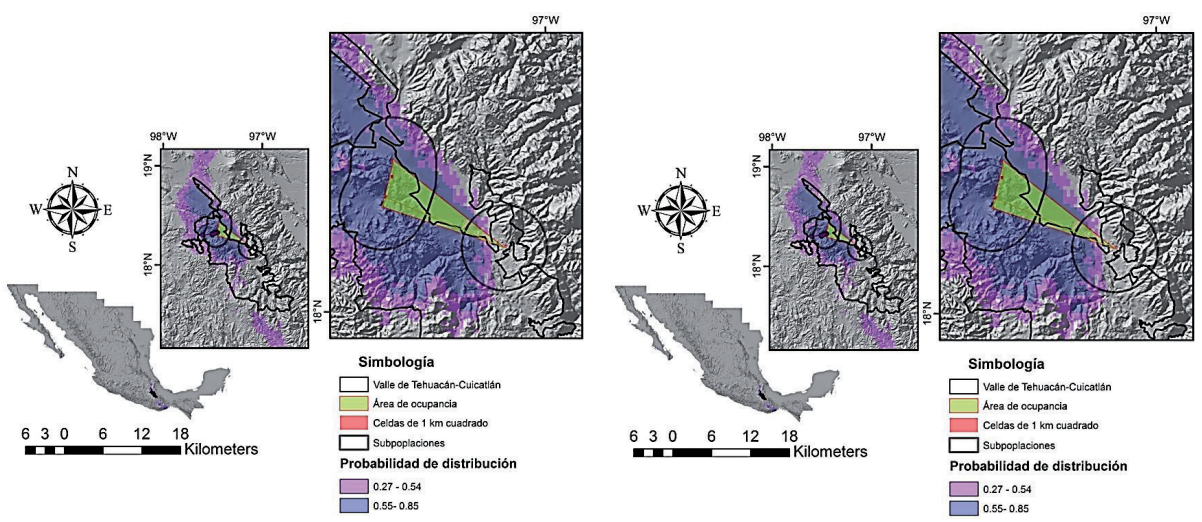

Fig. 5. Distribución geográfica potencial y Fig. 6. Distribución geográfica potencial áreas de ocupación de Agave marmorata. y áreas de ocupación de Agave peacockii. 
por los suelos del tipo LPq-RGc (Leptosol lítico-Regosol calcárico), ya que su distribución se situó principalmente en las áreas que comprende dicho tipo de suelo. Aunque también se localiza en diferentes tipos de suelo como el Regosol calcárico (RGc) y el Leptosol lítico (LPq). En lo que concierne a A. peacockii sólo se le pudo observar en suelos del tipo LPq-RGc (fig. 7). Cabe destacar que todos estos suelos presentan características buenas para el crecimiento radicular de estas especies, porque están favorecidas por la actividad microbiana, así como para el movimiento adecuado de nutrientes y agua, a pesar de que la materia orgánica presente en todos los tipos de suelo de la región es pobre (López-Galindo et al., 2003).

La vegetación que se asocia con $A$. marmorata corresponde al matorral crassicaule (MC), matorral espinoso (ME), matorral crassicaule-matorral subinerme (MC-MB), matorral espinoso-matorral crassicaule (ME-MC), vegetación secundaria (VS), agricultura de temporal (TA) y desmonte (D). Mientras que A. peacockii sólo se asocia con matorral crassicaule-matorral subinerme (MC-MB) (fig. 7).

El clima predominante en todas las zonas de estudio fue el BSohw el cual se caracteriza por ser seco. Este tipo de clima abarca toda la cuenca del río Salado y el área de Zapotitlán Salinas. Una característica importante es que la zona de estudio no presenta un rango de climas, lo cual determina el tipo de vegetación asociada a ambas especies de magueyes (fig. 8).

La altitud en la que se ubicaron a $A$. marmorata y A. peacockii fue de los 1417 a los 1560 m.s.n.m. (fig. 9).

En la figura 9, se observan los sitios y las pendientes donde se localizaron poblaciones de A. marmorata y A. peacockii. La pendiente predominante en los sitios de muestreo, generalmente, se ubicó en un rango de 0 a 25 grados. La orientación de ambas especies varió de un sitio a otro, debido a que se tomaron geoposiciones de manera azarosa.
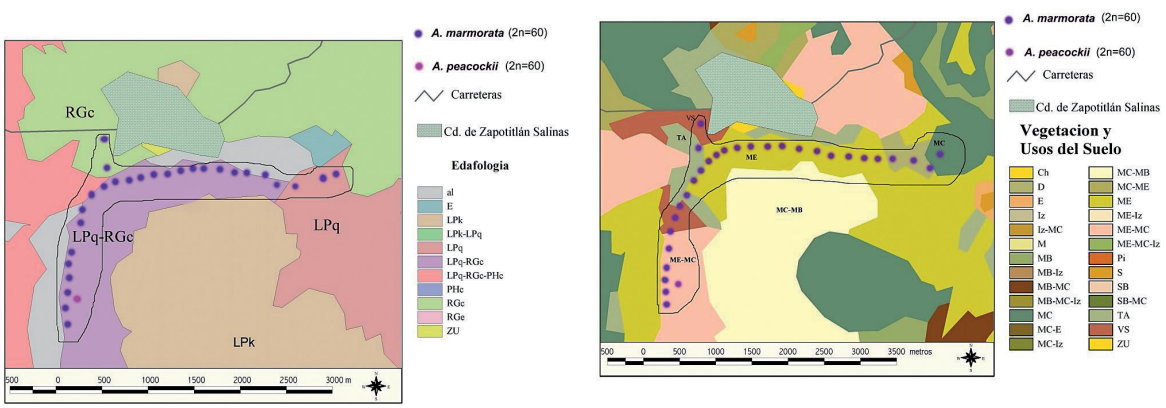

Fig. 7. Área de distribución en la zona de estudio de A. marmorata y A. peacockii en los tipos de suelo y tipos de vegetación. 


\section{DISCUSIÓN Y CONCLUSIONES}

Se registró por primera vez el número cromosómico de Agave marmorata y Agave peacockii $(2 \mathrm{n}=2 \mathrm{x}=60)$. En conclusión, el conteo cromosómico demostró que los niveles de ploidía de Agave marmorata y Agave peacockii corresponden a 2x. La expresión de su carácter diploide $(2 \mathrm{n}=2 \mathrm{x}$ $=60$ ) de estos agaves coincidió con lo que se señala en la literatura en relación con establecer a la altiplanicie mexicana como su centro de origen y distribución, debido a la presencia alta de agaves con carácter diploide en esta zona (Granick, 1944; Darlington, 1956; Cave, 1964; Banerjee y Sharma 1988, 1989).

Los resultados de esta investigación también corroboran los reportes sobre estudios citogenéticos y taxonómicos previos para la familia Agavaceae, asimismo los números cromosómicos reportados para las dos especies de agave son múltiplos del
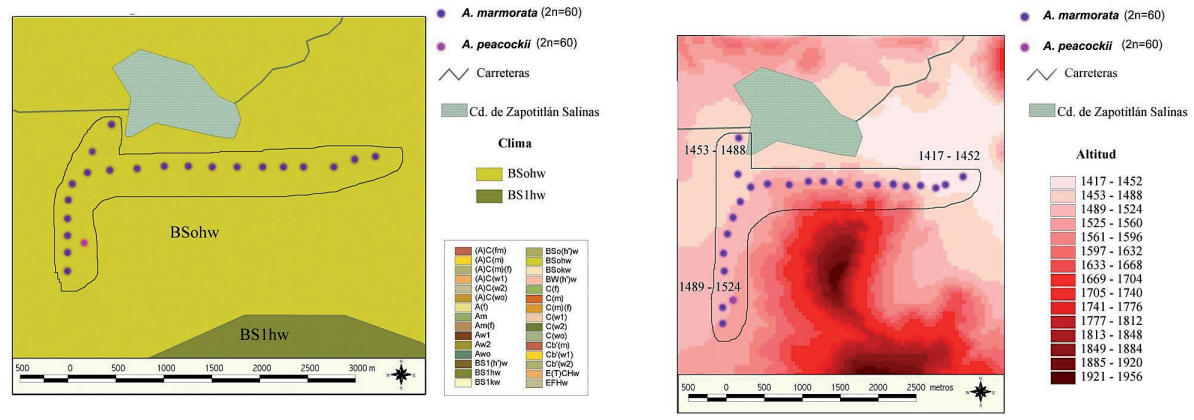

Fig. 8. Área de distribución en la zona de estudio de $A$. marmorata y A. peacockii en los tipos de altitud y clima.
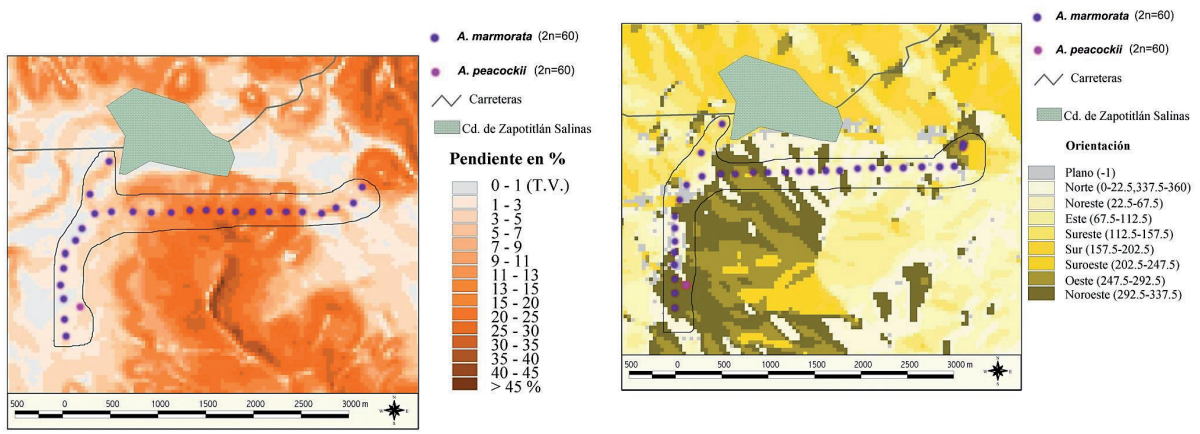

Fig. 9. Área de distribución en la zona de estudio de A. marmorata y A. peacockii en los tipos de pendiente y orientación. 
número básico establecido para el género $(\mathrm{x}=30)$; además ambas especies presentaron el carácter bimodal en sus cariotipos (10 cromosomas largos y 50 cromosomas pequeños en la condición diploide) y otro rasgo interesante descubierto en este estudio es la persistencia notable de los cromosomas grandes del tipo telocéntrico y subtelocéntrico (Granick, 1944; Cave, 1964; Castorena-Sánchez et al., 1991).

En el análisis del nivel de asimetría cariotípica para la relación entre los brazos cromosómicos (A1) se observó un valor alto en el nivel intracromosómico para ambas especies. Esto se debe a la presencia de un par cromosómico telocéntrico, cuatro pares subtelocéntricos y dos pares de cromosomas submetacéntricos en el genoma de $A$. peacockii. Para A. marmorata su complemento cromosómico posee cuatro pares de cromosomas subtelocéntricos y nueve pares de cromosomas clasificados como submetacéntricos. Esto último, se refleja en los valores promedio de sus índices centroméricos que fue de $38.10 \pm 10.1$ para $A$. marmorata y de $43.2 \pm 12.4$ para $A$. peacockii. Además, en el nivel de asimetría intercromosómica (A2) hay variaciones en la longitud de sus cromosomas ( 0.53 para A. marmorata y 0.85 para A. peacockii). Esta variación se observó mayor en la longitud de los cromosomas de A. marmorata, ya que como se señaló anteriormente la posición del centrómero varió en un $43 \%$, en cambio, $A$. peacockii tiene un porcentaje del $76 \%$ de cromosomas clasificados como estrictamente metacéntricos, es decir, en esta especie los cambios en la longitud de los cromosomas ha sido menor. En conclusión, esto es un indicativo de que los cariotipos de estas plantas son asimétricos de acuerdo a los criterios de Romero-Zarco (1986). Por tanto, esta condición asimétrica de los cariotipos puede ser tomada como un parámetro que indica la dirección evolutiva de las especies aquí analizadas, y hacen pensar que en relación a la especiación de este género y apoyando lo señalado por Castorena-Sánchez et al. (1991), quizás las mutaciones puntuales y los rearreglos estructurales de los cromosomas pequeños han tenido influencia en la evolución de $A$. marmorata y $A$. peacockii para su establecimiento y supervivencia en esta zona.

Este estudio también confirmó lo señalado por Brandham y Doherty (1998), en relación a que la familia Agavaceae presenta cariotipos del tipo de ortoselección; es decir, los miembros de esta familia presentan uniformidad en el número básico, su cariotipo es bimodal, y presentan similitudes en la longitud total de los cromosomas. Por tanto, A. marmorata y A. peacockii presentan cariotipos del tipo de ortoselección. Brandham y Doherty (1998) mencionaron que la familia Agavaceae al presentar cariotipos de ortoselección, sus tendencias evolutivas se deben principalmente al incremento de ácido desoxirribonucleico (DNA), que se distribuye en estos complementos cromosómicos en proporción a longitud del cromosoma, de manera que se conserva la uniformidad del cariotipo.

Existen reportes donde se ha observado variación en el número cromosómico somático en plantas con reproducción vegetativa (Banerjee y Sharma, 1988, 1989). Esto no es el caso en el presente estudio, a pesar de que estas dos especies en la subcuenca de Zapotitlán Salinas representaron poblaciones de origen asexual (clonal), no se reportan variaciones en el número cromosómico de estas especies, es decir, se registró una frecuencia muy baja en la variación 
cromosómica. En conclusión, el carácter diploide, la uniformidad de sus cariotipos y su forma endémica en estas especies puede acentuarse más y por consiguiente su sobrevivencia está en riesgo.

Por otra parte, de acuerdo con los resultados del área de ocupación obtenidos, en el estudio citogeográfico, se determinó que la distribución de $A$. marmorata en la localidad de Zapotitlán Salinas, Puebla es continua, estos valores indican distancias cortas entre individuos (tabla 3; figs. 7, 8 y 9); sin embargo, cabe destacar que estas distancias entre los individuos de este maguey se encuentran en un proceso de distanciamiento, esto se debe principalmente a la fuerte erosión y fragmentación que sufren los suelos de las terrazas aluviales, lo cual provoca que la especie en estudio se localice en áreas restringidas. A pesar de esto, esta especie tiene, según lo observado en el campo, presencia de organismos sexuales (presencia de inflorescencia), lo cual ayudaría a aumentar su distribución en la zona (fig. 5). Sin embargo, en la actualidad la transformación de las terrazas aluviales, el mal manejo de las especies, la extracción de estas especies de su hábitat y uso del suelo para actividades agrícolas afectan y amenazan el establecimiento y la distribución de estos magueyes en la zona.

Finalmente, al establecerse la relación entre los resultados del análisis citogenético y los de aerografía, para $A$. marmorata, se determina que la variación de las condiciones del suelo, los diferentes tipos de vegetación de la zona, así como el clima, no afectan el establecimiento y su distribución. En conclusión, la condición de diploide (2n) le dan la capacidad de poseer una respuesta mayor a los cambios ambientales naturales. Mien- tras que $A$. peacockii por su distribución geográfica limitada, su condición de ploidía (x), por su posible carácter híbrido y la presencia baja de individuos de origen sexual $y$ asexual su sobrevivencia se encuentra en mayor riesgo.

Nuestros resultados sirven como base para realizar estudios filogenéticos y biogeográficos futuros, que permitan generar aproximaciones más específicas que coadyuven a reconocer la condición endémica de estas especies.

\section{LITERATURA CITADA}

Álvarez de Zayas, A., 1989. "Distribución geográfica y posible origen de las agavaceas". Rev. Jard. Bot. Nac. (Cuba) Vol. X 1: 25-36.

Barrera, C.C., 2001. Descripción y regionalización fisiográfica del Valle de Zapotitlán, Puebla. Tesis de licenciatura, ENEP- Iztacala, UNAM. Tlalnepantla, Estado de México.

Banerjee, S., y A.K. Sharma, 1988. "Structural differences of chromosomes in diploid Agave". Cytologia, 53: 415-420.

Banerjee, S., y A. K. Sharma, 1989. "Structure and behavior of chromosomes in four different species of Agave". Cytologia, 54: 667-672.

Bhattacharyya, G.N., 1968. "Chomosomes in different species of Agave". J. Cyt. Genet., 3: 1-6.

Brandham, P.E., y M.J. Doherty, 1998. "Genome size variation in Aloaceae, 
an angiosperm family displaying karyotypic orthoselection". An. Bot., 82 (Suppl. A): 67-73.

Castorena-Sánchez, I., 1985. Estudio citogenéticos en agaves productores de fibra en Yucatán. Tesis de licenciatura. Facultad de Ciencias, UNAM. México.

Castorena-Sánchez, I.; R.M. Escobedo, y A. Quiroz, 1991. "New cytotaxonomical determinants recognized in six taxa of Agave in the sections Rigidae and Sisalanae". Can. J. Bot., 69: 127-1264.

Cave, S. M., 1964. "Cytological observations on some genera of the Agavaceae". Madroño, 17: 163-169.

CONABIO (Comisión Nacional para el Conocimiento y uso de la Biodiversidad), 2012. Lista de especies vegetales protegidas, términos de referencia generales.[htpp://www.conabio.gob. $\mathrm{mx}$ ] (accesado 23 de febrero 2013).

Darlington, C.D., 1956. Chromosome Botany. George Allen \& Unwin, London ESRI (Environmental Scientific Research Institute), 2000. ArcView 3.2. ESRI, Redlans.

Flores-Maya S.; J.C. Moreno-Ramírez, S. Romero-Rangel, y C. Rojas-Zenteno, 2010. Análisis morfológico y cariológico de Agave karwinskii Zucc. y Agave macroacantha Zucc. en el municipio de Zapotitlán Salinas, Puebla. Biocyt, 3(11): 181-196.

Galindo, F.L.; I.D. Muñoz, M.H. Moreno, A.A. Soler, y G.H. Puga, 2007. "Eva- luación del Ambiente Físico de las Terrazas Fluviales y Valle de Zapotitlán". Arizmendi, M.D. et al. (eds.), Deterioro Ambiental en Zonas Aridas. Facultad de Estudios Superiores Iztacala-UNAM-UBIPRO.

García, V.A., 1990. Técnicas y procedimientos de citogenética vegetal. 3a ed. Colegio de postgraduados. México.

García, M.G., 2001. Mapeo y caracterización de las Terrazas Aluviales del Valle de Zapotitlán de las Salinas, Puebla. Tesis de licenciatura. FES Iztacala. UNAM. México.

García-Mendoza, A., 1995. "Riqueza y Endemismo de la familia Agavaceae en México". En: Linares, E., Dávila, P. Chiang, F., Bye, R. y Elias, T.S. (eds.) Conservación de plantas en peligro de extinción: diferentes enfoques. UNAM. México.

2002. "Distribution of Agave (Agavaceae) in Mexico". Cac. Suc. J., 74: 177-186.

García-Mendoza, A., 2007. "Los agaves de México”. Ciencias, 87: 14-23.

Gentry, H.S., 1982. Agaves of continental North America. University of Arizona. USA.

Granick, B.E., 1944. "A Karyosistematic study of the genus Agave". Am. J. Bot., 31: 283-298.

IPCN (Index to plant Chromosome numbers), 2013. Base de datos de números cromosómicos en plantas. [htpp:// 
mbot.org] (accesado 12 de febrero 2013).

INEGI (Instituto Nacional de Estadística Geografía e Informática), 1987. Síntesis geográfica, nomenclator y anexo cartográfico del estado de Puebla. México.

, 2005. Conjunto de Datos Vectoriales de Uso de Suelo y Vegetación. Escala 1:250,000, Serie III (Conjunto Nacional). Dirección General de Geografía, Instituto Nacional de Estadística, Geografía e Informática, México.

Levan, A.K.F. y A.A. Sandberg, 1964. "Nomenclature for centromeric position on chromosomes". Hereditas, 52: 20-220.

López-Galindo, F.; D. Muñóz-Iniestra, M. Hernández-Moreno, A. Soler-Aburto, M.C., Castillo-López, y I. Hernández-Arzate, 2003. "Análisis integral de la toposecuencia y su influencia en la distribución de la vegetación y la degradación del suelo en la Subcuenca de Zapotitlán Salinas, Puebla”. Bol. Soc. Geol. Mex., 1: 19-41.

Martínez-Palacios, A.; L.E. Eguiarte, y R. G. Furnier, 1999. "Genetic diversity of the endangered endemic Agave victoriae-reginae (Agavaceae) in the Chihuahua desert". Am. J. Bot., 86: 1093-1098.

Oliveros-Galindo, 2000. Descripción estructural de las comunidades vegetales en las terrazas fluviales del rio el Salado, en el valle de Zapotitlán de la Salinas, Puebla, México. Tesis de licenciatura. Facultad de Estudios Profesionales Iztacala, Universidad
Nacional Autónoma de México. Tlalnepantla, Edo. de México.

Pinkava, D.J., y M.A. Baker, 1985. "Chromosome and hybridization studies of agaves". Desert Plants, 7: 93-100.

Pinkava, D.J.; B.D. Parfitt, M.A. Baker, y R.D.Worthington, 1992. "Chromosome numbers in some cacti of western north America VI, with nomenclatural changes". Madroño, 39: 98-113.

Rapoport, E.H., 1975. Areografia. Estrategias Geográficas de las Especies. Fondo de Cultura Económica. México.

Reinchenbacher, F., 1985. "Conservation of Southwestern Agaves". Desert Plant, 7(88): 103-106 .

Rodríguez, V.M., y G. Seijo, 2001. “Estudio del ciclo celular en una especie leñosa: Gleditsia amorphoides (Leguminosae)". Facultad de Ciencias Agrarias, 4.

Romero, Z.C., 1986. "A new method for estimating karyotipe asymmetry". Taxon, 35: 526-530.

Rzedowski, J., 1978. Vegetación de México. Limusa. México.

SEMARNAT (Secretaría de Medio Ambiente y Recursos Naturales), 2005. CONANP. (Áreas Naturales Protegidas), 2012. Reserva de la biosfera Tehuacán-Cuicatlán. [htpp://www.semarnat. gob.mx].(accesado 4 de marzo 2013).

Sokal, R.R., y F. J. Rohlf, 1995. Biometry. W. H. Freeman and Company, New York. 
Suárez-Mota, M.E., y J.L. Villaseñor, 2011. "Las compuestas endémicas de Oaxaca, México: diversidad y distribución". Bol. Soc. Bot. Méx., 88: 55-66.

Suárez-Mota M.E.; O. Téllez-Valdés, R. Lira-Saade, y J.L. Villaseñor, 2013. "Una regionalización de la Faja Volcánica Transmexicana con base en su riqueza florística". Bot. Scie., 91(1): En prensa.

UICN., 2001. Categorías y criterios de la lista Roja de la UICN. Versión 3.1. Comisión de Supervivencia de Especies de la UICN (Unión Internacional para la Conservación de la Naturaleza). Cambridge.

UTHSCSA (The University of Texas Health Science Center at San Antonio), 2012. Analizador de imágenes versión 3.1. [http://www.uthscsa.com]. (accesado 22 de septiembre 2012).

Valiente-Banuet, A.; P.M.C. Dávila Arizmendi, A. Rojas-Martínez, y A. Casas,
1995. "Bases ecológicas del desarrollo sustentable en zonas áridas: el caso de los bosques de cactáceas columnares del Valle de Tehuacán y Baja California Sur, México". En: IV Curso sobre desertificación y desarrollo sustentable en América Latina y el Caribe. Colegio de Posgraduados, Edo. de Mexico.

Valverde, P.L.; F. Vite, y I. Zavala-Hurtado, 1996. "A morphometric analysis of a putative hybrid between Agave marmorata Roezl and Agave kerchovei Lem. Agave peacockii Croucher". Bot. J. Linn. Soc., 122: 155-161.

Vázquez, R.A., 1977. Estudio citogenético y de variación en una población de $A g a-$ ve atrovierens. Tesis de licenciatura. Facultad de Ciencias, UNAM. México.

Zavala-Hurtado, J.A., 1982. "Estudios ecológicos en al Valle Semiárido de Zapotitlán, Puebla. I. Clasificación numérica de la vegetación basada en atributos binarios de presencia o ausencia de las especies". Biotica, 7: 97-120.

Recibido: 23 de mayo de 2013. Aceptado: 15 de enero de 2015. 